\title{
Date of Tumor Progression After Initial Treatment
}

National Cancer Institute

\section{Source}

National Cancer Institute. Date of Tumor Progression After Initial Treatment. NCI

Thesaurus. Code C158627.

The data of tumor progression after initial treatment. 